\title{
Indicadores de calidad para evaluar buenas prácticas docentes de «mobile learning» en Educación Superior
}

\section{Quality indicators to evaluate good teaching practices of mobile learning in Higher Education}

Inmaculada Aznar Díaz, María Pilar Cáceres Reche, José María Romero Rodríguez

Universidad de Granada, Granada, España

\{iaznar, caceres, romejo\}@ugr.es

http://orcid.org/0000-0002-0018-1150

http://orcid.org/0000-0002-6323-8054

http://orcid.org/0000-0002-9284-8919

\section{Resumen}

El mobile learning se alza como una metodología emergente que ya se está aplicando en distintas etapas educativas, por tanto es necesario poner el foco de atención en el tipo de prácticas que se están implementando con dispositivos móviles. El objetivo de este trabajo ha sido establecer un sistema de indicadores de calidad para evaluar buenas prácticas de mobile learning en educación superior. Para ello, se ha seguido un proceso riguroso en la confección de cada uno de los indicadores, los cuales han sido validados mediante la técnica del juicio de expertos. como resultado principal se establece un sistema de 25 indicadores, agrupados en cinco variables, para su implementación en contextos educativos universitarios donde se aplique la metodología mobile learning. En suma, el sistema expuesto sirve de modelo de referencia para discernir entre la simple introducción de los dispositivos móviles, de su uso planificado y con carácter pedagógico.

\section{Keywords}

Indicadores de calidad; Mobile Learning; Buenas prácticas docentes; Educación superior

\begin{abstract}
Mobile learning stands as an emerging methodology that is already being applied in different educational stages, so it is necessary to focus on the type of practices that are being implemented with mobile devices. The objective of this paper has been to establish a system of quality indicators to evaluate good practices of mobile learning in Higher Education. For this, a rigorous process has been followed in the preparation of each of the indicators, which has been validated by the technique of expert judgment. As a main result, a system of 25 indicators is established, grouped into 5 variables, to be implemented in university educational contexts where the mobile learning methodology is applied. In summary, the exposed system serves as a reference model to discern between the simple introduction of mobile devices for their planned use and with pedagogical character.
\end{abstract}

\section{Palabras clave}

Quality Indicators; Mobile Learning; Good Teaching Practices; Higher Education

\section{Introduction}

Una parte fundamental para comprobar los resultados de la aplicación de un elemento en cualquier contexto es la evaluación. Y, tal y como resalta García-Valcárcel y Tejedor (2010), es necesaria la evaluación de las experiencias que se vienen desarrollando con Tecnologías de la Información y la Comunicación (TIC). El establecimiento de indicadores de calidad para evaluar prácticas educativas con TIC permite poner el foco en ciertos ítems a los que habrá que prestar especial atención en 
el proceso evaluativo. En esta línea se encuentra Cardoso y Cerecedo (2011), quienes establecen una serie de indicadores para acreditar la calidad de un programa de posgrado en educación; Gutiérrez, Rodríguez y Pantoja (2014), que utilizan una lista de indicadores de calidad para evaluar la incorporación de las TIC en programas formativos; y Nolasco y Ojeda (2016), que establecen 58 indicadores de calidad para la evaluación de las TIC a través de la adaptación de los indicadores TIC de la metodología UNESCO.

Resulta, pues, imprescindible contar con un marco constituido por una serie de indicadores de calidad que saquen a relucir aquellos aspectos clave para la determinación de una buena práctica y evaluación de las TIC, validados a partir de técnicas como el juicio de expertos (Cabero y Barroso, 2013).

Particularmente, en este trabajo se ha puesto el foco de atención en la introducción didáctica de los dispositivos móviles en el aprendizaje, denominándose esta metodología docente con el concepto mobile learning o su traducción al español, aprendizaje móvil. Brazuelo y Gallego (2011, p. 17) definen este término como "la modalidad educativa que facilita la construcción del conocimiento, la resolución de problemas de aprendizaje y el desarrollo de destrezas o habilidades diversas de forma autónoma y ubicua gracias a la mediación de dispositivos móviles portátiles". De forma simplificada el mobile learning se corresponde con la mediación de los dispositivos móviles para el desarrollo del proceso de enseñanza-aprendizaje (Aznar, Romero y Rodríguez-García, 2018).

En el contexto de la educación superior la implementación del mobile learning está obteniendo un mayor desarrollo que en otras etapas educativas (Wu et al., 2012). El uso normalizado de los dispositivos móviles por parte de los estudiantes y la libertad para portarlo a cualquier lugar, hacen que sea más sencilla su introducción en el contexto universitario. Además, la producción científica sobre mobile learning no para de incrementarse en los últimos años, con especial énfasis en la educación superior (Mateus, Aran-Ramspott y Masanet, 2017). Este hecho conlleva ciertas ventajas relacionadas principalmente con la ubicuidad, que permite la facilidad, rapidez y comodidad de acceder a los contenidos (Grané, Crescenzi y Olmedo, 2013); sin contar los múltiples beneficios en la mejora del rendimiento académico de los estudiantes (Jeno, Grytnes y Vandvik, 2017) y el desarrollo de habilidades como el trabajo cooperativo (Kearney et al., 2012; Monguillot et al., 2014), competencia digital (Ramos, Herrera y Ramírez, 2010; Suárez, Lloret y Mengual, 2016) y autorregulación del aprendizaje (Suárez, Crescenzi y Grané, 2013; Sevillano y Vázquez, 2015). No obstante, su implementación no está exenta de algunas limitaciones derivadas de la formación docente y los recursos institucionales (Ramírez y García, 2017).

En este escenario incipiente de introducción de la tecnología móvil en la educación superior, se hace necesario el establecimiento de criterios de calidad para la evaluación de las prácticas que se están desarrollando con dispositivos móviles (Caldeiro, Yot y Castro, 2018). En primera instancia, para 
poder discernir una práctica didáctico-pedagógica, fundamentada en una metodología docente, de la simple aplicación de un recurso puntual sin ningún fin educativo. En segunda instancia, para contar con modelos de buenas prácticas docentes que sirvan de referente. En última instancia, con el fin de contribuir y seguir formando un cuerpo sólido de conocimientos sobre la implementación de los dispositivos móviles para la mejora del aprendizaje.

Así pues, el establecimiento de los indicadores de calidad parte de un sustento teórico consolidado sobre el mobile learning, de modo que se han tenido en cuenta diversas aportaciones teóricas sobre el estado del arte (Brazuelo y Gallego, 2011; Kearney et al., 2012; Herrera, Sanz y Fennema, 2013; Ramírez-Montoya y García-Peñalvo, 2017) y empíricas, tales como experiencias y resultados de investigaciones (Ramos, Herrera y Ramírez, 2010; Suárez, Crescenzi y Grané, 2013; Monguillot et al., 2014; Sevillano y Vázquez, 2015; Suárez, Lloret y Mengual, 2016; Jeno, Grytnes y Vandvik, 2017; Fernández, 2018).

Por tanto, considerando el cuerpo teórico de conocimiento que se ha generado en los últimos años sobre el mobile learning y a la vista de estudios previos como el marco para el Análisis, Diseño y Evaluación de Experiencias y proyectos de mobile learning (MADE-mlearn) -que propone cinco categorías de análisis: infraestructura, interacción, contenido, fundamentos de enseñanza y aprendizaje, y resultados obtenidos (Herrera, Sanz y Fennema, 2013)-; del modelo pedagógico de aprendizaje móvil de Kearney et al. (2012); y de las contribuciones anteriormente citadas, se ha establecido la definición de calidad en las prácticas de mobile learning, la cual hace referencia a: la utilización de los dispositivos móviles con la finalidad de favorecer la construcción del conocimiento y producir un aprendizaje significativo a partir de la autorregulación, el trabajo cooperativo y el desarrollo de la competencia digital.

En suma, la evaluación de las prácticas con dispositivos móviles forma parte del proceso para determinar si nos encontramos con una actividad caracterizada por ser una buena práctica docente con TIC, entendiendo este término como la actividad que emplea la tecnología y conlleva resultados satisfactorios (implicación, motivación, desarrollo de habilidades), convirtiéndose en prácticas transferibles a otros contextos por su excelencia (Fernández y Torres, 2015).

\subsection{Proceso de elaboración de los indicadores de calidad de mobile learning}

A partir de la definición de calidad en las prácticas de mobile learning, se han establecido las distintas variables que han servido de referente para elaborar los indicadores. Estas se han seleccionado por vía deductiva en base a la consideración teórica y/o empírica de diferentes estudios, los cuales relacionan directamente la implicación de estas variables con el mobile learning: 
- Dispositivos móviles (V1) (Brazuelo y Gallego, 2011; Ramírez y García, 2017): hace referencia a aquellos aspectos relativos al uso de los dispositivos móviles (smartphones, tablets) con los que se lleva a cabo el proceso de aprendizaje.

- Competencia digital (V2) (Ramos, Herrera y Ramírez, 2010; Suárez, Lloret y Mengual, 2016): disposición de habilidades para saber discriminar la información disponible en la red, comunicarse a través de redes digitales, crear contenido digital y resolver eficazmente problemas con el uso de la tecnología, al mismo tiempo que se realiza un uso responsable y seguro de la red.

- Construcción del conocimiento (V3) (Brazueloy Gallego, 2011; Sevillano y Vázquez, 2015; Fernández, 2018): creación de nuevo conocimiento por parte del estudiante de forma comprensiva a partir de la información existente, conocimientos previos, experiencia e interacción con el entorno.

- Autorregulación del aprendizaje (V4) (Suárez, Crescenzi y Grané, 2013; Sevillano y Vázquez, 2015; Jeno, Grytnes y Vandvik, 2017): proceso en el cual el estudiante forma parte activa de su aprendizaje a través de la reflexión y toma de conciencia sobre cómo aprende, puesto que es él mismo el que establece las pautas para aprender, tiempos para ello, estrategias y motivación. De tal modo que adapta el aprendizaje a sus necesidades y actúa en base a ellas.

- Trabajo cooperativo (V5) (Kearney et al., 2012; Monguillot et al., 2014): modo de actuar de forma autónoma y conjunta entre los miembros de un grupo con la finalidad de realizar una tarea colectiva y aprender unos de otros.

Con el fin de asegurar la propia calidad de los indicadores, se han seguido los criterios de selección de la guía establecida por el Departamento Administrativo Nacional de Estadística de Colombia (DANE, 2009) sobre el diseño, construcción e interpretación de indicadores (tabla 1).

\begin{tabular}{|c|l|}
\hline Criterios & \multicolumn{1}{|c|}{ Constatación en los indicadores } \\
\hline Pertinencia & $\begin{array}{l}\text { Los indicadores expresan de forma clara que se pretende medir } \\
\text { describiendo adecuadamente la situación a observar. }\end{array}$ \\
\hline Funcionalidad & Los indicadores son operacionales, es decir, son medibles. \\
\hline Disponibilidad & $\begin{array}{l}\text { La información que resaltan los indicadores es estable, puede } \\
\text { consultarse en cualquier momento. }\end{array}$ \\
\hline Confiabilidad & $\begin{array}{l}\text { Los datos que se obtienen de los indicadores pueden traducirse } \\
\text { en números, por lo que presentan atributos de calidad estadística. }\end{array}$ \\
\hline Utilidad & $\begin{array}{l}\text { Los resultados extraídos del cómputo de indicadores de una misma } \\
\text { variable permiten tomar decisiones acerca de si esa variable está } \\
\text { presente en las prácticas de mobile learning o por el contrario } \\
\text { requiere una mejora. }\end{array}$ \\
\hline
\end{tabular}

Tabla 1. Criterios que cumplen los indicadores de calidad en base a DANE (2009) 
Del mismo modo, se han tenido en cuenta los criterios relacionados con la utilidad y comprensión de los indicadores (DANE, 2009) (tabla 2).

\begin{tabular}{|c|l|}
\hline Criterios & \multicolumn{1}{|c|}{ Constatación en los indicadores } \\
\hline Aplicabilidad & $\begin{array}{l}\text { Los indicadores responden a una necesidad real, establecida en } \\
\text { la definición de calidad extraída de planteamientos teóricos y } \\
\text { experiencias empíricas. }\end{array}$ \\
\hline No redundancia & $\begin{array}{l}\text { Cada indicador expresa por sí mismo la acción a observar, } \\
\text { sin solaparse con ningún otro. De tal manera que no hay dos } \\
\text { indicadores que midan el mismo factor. }\end{array}$ \\
\hline Interpretabilidad & $\begin{array}{l}\text { Los indicadores están diseñados y formulados de forma sencilla, } \\
\text { lo que facilita su entendimiento tanto por expertos como por } \\
\text { cualquier otra persona. }\end{array}$ \\
\hline Comparabilidad & $\begin{array}{l}\text { Los distintos indicadores son generalizables, de modo que } \\
\text { pueden compararse entre ellos mismos aun habiendo recogido } \\
\text { los datos en otros contextos. }\end{array}$ \\
\hline Oportunidad & $\begin{array}{l}\text { Los datos que se obtienen son inmediatos, lo que facilita } \\
\text { reajustar el proceso de aprendizaje para la consecución de los } \\
\text { objetivos. }\end{array}$ \\
\hline
\end{tabular}

Tabla 2. Criterios de utilidad y comprensión que cumplen los indicadores de calidad en base a DANE (2009)

Teniendo en cuenta los diferentes criterios en los que se basan los indicadores y en la propia definición de calidad aportada, podemos afirmar que se sigue un proceso riguroso en su elaboración, que deriva, por tanto, en un sistema de categorías válido para su aplicación en contextos donde se pretenda determinar si una experiencia de mobile learning puede ser considerada como buena práctica.

\section{Métodos de validación}

Los indicadores de calidad han sido sometidos a un juicio de expertos para determinar la validez de contenido (Escobar y Cuervo, 2008). De modo que se invitó a un grupo de expertos especialistas en TIC para que emitieran un juicio sobre los diferentes indicadores establecidos y su adecuación en función de la variable donde se engloba cada uno de ellos.

Así pues, definiendo la estrategia del juicio de expertos como la opinión de aquellas personas especialistas en una determinada temática que dan su veredicto acerca de la cuestión que se les plantea, en palabras de Robles y Rojas (2015, p. 2), sus funciones principales son "eliminar aspectos irrelevantes, incorporar los que son imprescindibles y/o modificar aquellos que lo requieran”.

En cuanto a la selección de los expertos se aplicaron una serie de criterios:

Conocimiento sobre el objeto de estudio. 
- $\quad$ Experiencia docente en educación superior.

- $\quad$ Aplicación de tecnología en las materias que imparte.

- $\quad$ Producción de literatura científica relacionada con las TIC.

Finalmente participaron un total de seis expertos que cumplían dichos criterios, suficiente debido al objeto de estudio en base a las consideraciones de Cabero y Llorente (2013) que destacan que no hay acuerdo unánime en el número óptimo de expertos, el cual depende principalmente de la accesibilidad a los mismos. Estos expertos se caracterizan por aplicar metodologías basadas en las TIC en la docencia y, en su mayoría, por tener una experiencia consolidada en educación superior de más 10 años. Además de conocer el objeto de estudio, publicar artículos científicos y participar en congresos nacionales e internacionales sobre tecnología educativa.

Por otro lado, la técnica de validación empleada fue individual, obteniendo información de cada uno sin que estuvieran en contacto entre ellos. El sistema que se siguió consistió en la elaboración de un cuestionario ad hoc on-line que recogía la definición de calidad sobre buenas prácticas en mobile learning para, posteriormente, preguntar acerca de la pertinencia de las variables extraídas de la definición y los indicadores asociados a cada una de ellas en base a tres niveles de respuesta - sí; no; lo modificaría)-. Por ende, se demandó a los expertos que valoraran cualitativamente los diferentes ítems en cuanto a su construcción y definición.

\section{Resultados}

Las respuestas obtenidas de los expertos consultados muestran unanimidad en la adecuación de la mayor parte de los ítems propuestos, asimismo el porcentaje de acuerdo de cada uno de ellos se sitúa en valores positivos de concordancia entre expertos (tabla 3).

\begin{tabular}{|c|c|}
\hline Ítems & Porcentaje de acuerdo (\%) \\
\hline Variable 1 & 100,00 \\
\hline Indicador 1-1 & 66,66 \\
\hline Indicador 1-2 & 83,33 \\
\hline Indicador 1-3 & 83,33 \\
\hline Indicador 1-4 & 83,33 \\
\hline Indicador 1-5 & 83,33 \\
\hline Variable 2 & 83,33 \\
\hline Indicador 2-1 & 100,00 \\
\hline Indicador 2-2 & 100,00 \\
\hline Indicador 2-3 & 100,00 \\
\hline Indicador 2-4 & 100,00 \\
\hline Indicador 2-5 & 100,00 \\
\hline
\end{tabular}




\begin{tabular}{|c|c|}
\hline Indicador 2-6 & 100,00 \\
\hline Variable 3 & 83,33 \\
\hline Indicador 3-1 & 100,00 \\
\hline Indicador 3-2 & 83,33 \\
\hline Indicador 3-3 & 100,00 \\
\hline Indicador 3-4 & 83,33 \\
\hline Variable 4 & 83,33 \\
\hline Indicador 4-1 & 83,33 \\
\hline Indicador 4-2 & 83,33 \\
\hline Indicador 4-3 & 83,33 \\
\hline Indicador 4-4 & 83,33 \\
\hline Variable 5 & 100,00 \\
\hline Indicador 5-1 & 66,66 \\
\hline Indicador 5-2 & 100,00 \\
\hline Indicador 5-3 & 100,00 \\
\hline Indicador 5-4 & 100,00 \\
\hline Indicador 5-5 & 100,00 \\
\hline Indicador 5-6 & 100,00 \\
\hline
\end{tabular}

Tabla 3. Porcentaje de acuerdo por cada item consultado

En cuanto a las respuestas concretas:

Experto 1 (mujer, 12 años de experiencia en educación superior) cree pertinentes la totalidad de indicadores y sugiere la modificación de los indicadores 1-1, 1-3, 1-4, 3-2, 4-4 y 5-1. Además, propone incluir algunos aspectos en las definiciones de las variables competencia digital (V2), construcción del conocimiento (V3), autorregulación del aprendizaje (V4), y trabajo cooperativo (V5).

Experto 2 (hombre, 11 años de experiencia en educación superior) y experto 3 (mujer, 15 años de experiencia en educación superior) confirman la pertinencia de la totalidad de indicadores y no sugieren ninguna modificación en la definición de las variables ni en la formulación de los indicadores.

Experto 4 (mujer, 18 años de experiencia en educación superior) cree adecuados la totalidad de los indicadores y sugiere modificaciones en el indicador 3-4.

Experto 5 (mujer, 3 años de experiencia en educación superior) considera pertinentes la totalidad de los indicadores y sugiere modificaciones en los indicadores 1-3, 1-5, 2-1 y 2-3. Del mismo modo sugiere añadir algunas cuestiones en la variable 3. Por último, el experto 6 (hombre, 11 años de experiencia en educación superior) difiere de todos los demás proponiendo la eliminación de los indicadores 1-1, 1-2, 4-3 y 5-1. Además de la modificación de 1-5 y 4-2. Respecto a las sugerencias que realizan:

Indicador 1-1. Los estudiantes disponen de dispositivos móviles.

Tendría en cuenta el contexto y características del grupo (edad, nivel socioeconómico, etapa educativa), en el que se va a desarrollar el estudio, indicaría si se trata de "educación superior", del barrio o entorno 
de ubicación, país, etc. pueden o no disponer de dispositivos móviles (brecha digital).

Indicador 1-3. Los estudiantes utilizan únicamente los dispositivos móviles en el tiempo destinado a su aplicación en el aula.

Sería interesante plantearse cómo se controlaría eso, esto es, que el docente sepa que realmente se está trabajando la actividad indicada y no haciendo otro tipo de consultas.

Debe modificarse un poco el lenguaje. Para aquellos que nos especializamos en tecnología educativa puede ser que lo comprendamos, pero si lo enfocamos hacia aquellos que no, puede conllevar cierto sesgo debido a que no sepan bien qué responder. Además, los datos de multitud de estudios afirman que los dispositivos móviles se utilizan más por entretenimiento y ocio que por aprendizaje.

Indicador 1-4. Los docentes planifican los recursos a utilizar mediante los dispositivos móviles y establecen pautas para su uso.

Quizá planificación y "control temporal" de la tarea para evitar precisamente lo que comentaba anteriormente respecto al uso inadecuado del dispositivo móvil para otros fines.

Indicador 1-5. Los docentes saben manejar adecuadamente los dispositivos móviles.

Hay que tener en cuenta que a nivel instrumental la mayoría de los individuos sabemos tener un manejo de los dispositivos móviles. Quizá no es tanto el dispositivo, sino conocer el uso pedagógico que puede hacerse de él. Añadiría "pedagógico o didáctico" para contextualizarlo.

Concretar en mayor grado los tipos de uso.

Variable 2. Competencia digital.

Incluiría una habilidad denominada "metatecnología", esto es, plantearse de forma consciente, reflexiva y crítica el uso e impacto de las TIC en la educación, valores y modelos sociales (información que nos llega, redes sociales, identidad digital, etc.).

Indicador 2-1. Los estudiantes saben discriminar adecuadamente la información relevante disponible en la red.

Añadiría "buscar", aunque para evitar confusiones sería un ítem aparte. Uno de los principales escollos que tienen que superar los estudiante es, precisamente, saber acceder a la red para la búsqueda de información y luego discriminarla.

Indicador 2-3. Los estudiantes son capaces de comunicar y compartir la información socialmente a través de los dispositivos móviles. 
Una duda, ¿a nivel formativo? ¿a nivel social? ¿a nivel laboral? ¿a nivel de entretenimiento?

Variable 3. Construcción del conocimiento.

Incluiría además de "forma comprensiva".

Añadiría el término ubicuidad o ubicuo, ya que una de las características de los dispositivos móviles es que nos permiten aprender, compartir, relacionarlos, comunicarnos, etc. más allá del aula, tanto a docentes como a estudiantes, por lo que dota de ubicuidad al proceso de enseñanza-aprendizaje.

Indicador 3-2. Los estudiantes son capaces de generar nuevo conocimiento.

Añadiría también la "transferencia a la realidad": generar nuevo conocimiento con aplicabilidad práctica.

Indicador 3-4. Se establece un feedback entre el docente y el alumnado en las diferentes tareas.

Incluiría el alumnado entre sí.

Variable 4. Autorregulación del aprendizaje.

Incluiría la "reflexión y toma de conciencia sobre cómo aprende, esto es, autonocimiento que implicaría el autocontrol o autorregulación", serían habilidades metacognitivas. No se pueden marcar pautas para aprender si previamente no se ha realizado una reflexión constante sobre fortalezas, debilidades, limitaciones y cómo intervenir para mejorar.

Indicador 4-2. Las actividades favorecen la autorregulación del aprendizaje.

Yo no hablaría solo de actividades. Incluiría desarrollo de tareas, proyectos.

Indicador 4-4. Los estudiantes consideran que el «mobile learning» les ayuda a autorregular su aprendizaje.

Quizás, sería importante considerar además de esta metodología y sus potencialidades, la interacción que el alumno haga con ella y ahí tiene un gran papel el docente: cómo medie en crear un clima de clase motivante, que potencie la toma de conciencia con el uso de estos dispositivos y que, a su vez, repercuta en un aprendizaje autorregulado. Creo que solamente la metodología por si sola sería insuficiente para canalizar y desarrollar este constructo.

Variable 5. Trabajo cooperativo.

Precisar un poco más en cuanto a posibles semejanzas con "aprendizaje colaborativo".

Indicador 5-1. La disposición del mobiliario del aula favorece la cooperación entre los estudiantes. 
¿Cooperación y colaboración se trabajan de forma simultánea en el estudio? ¿Se entienden en este caso como sinónimos? Sería importante clarificar y ver hacia qué tendencia apunta o interesa en este estudio.

En suma, se han atendido todas las sugerencias por parte de los expertos e implementado aquellas en las que coincide la mayoría, teniendo en cuenta principalmente las que se encuentran fundamentadas en la literatura científica como características propias del mobile learning y no se solapan con otros indicadores de calidad. Así pues, se han efectuado las consideraciones del experto 5 y 6 sobre el indicador 1-5 en relación a considerar el carácter "pedagógico o didáctico", para matizar el ítem y así atender ambas propuestas de los dos expertos. Por otro lado, también se han añadido algunas de las sugerencias del experto 1 sobre la inclusión del concepto "comprensiva" en la definición de la variable 3, "reflexión y toma de conciencia sobre cómo aprende" en la variable 4 y la precisión comentada sobre el indicador 5-1 acerca de discernir si se trataba de aprendizaje cooperativo o colaborativo, de modo que se ha matizado.

\section{Sistema de indicadores de calidad para evaluar buenas prácticas con mobile learning}

A continuación, se detalla el sistema de indicadores de calidad para evaluar buenas prácticas en mobile learning: dispositivos móviles (tabla 4); competencia digital (tabla 5); construcción del conocimiento (tabla 6); autorregulación del aprendizaje (tabla 7); y trabajo cooperativo (tabla 8).

\begin{tabular}{|l|}
\hline \multicolumn{1}{|c|}{ Indicadores V1. Dispositivos móviles } \\
\hline 1-1. Los estudiantes disponen de dispositivos móviles. \\
\hline 1-2. Existe conexión a internet en el aula. \\
\hline 1-3. Los estudiantes utilizan únicamente los dispositivos móviles en el tiempo destinado a su aplicación en el aula. \\
\hline 1-4. Los docentes planifican los recursos a utilizar mediante los dispositivos móviles y establecen pautas para su uso. \\
\hline 1-5. Los docentes saben manejar adecuadamente los dispositivos móviles. \\
\hline
\end{tabular}

Tabla 4. Indicadores de calidad asociados a la variable dispositivos móviles

\section{Indicadores V2. Competencia digital}

2-1. Los estudiantes saben discriminar adecuadamente la información relevante disponible en la red.

2-2. Los estudiantes saben producir contenido digital utilizando los dispositivos móviles.

2-3. Los estudiantes son capaces de comunicar y compartir la información socialmente a través de los dispositivos móviles.

2-4. Los estudiantes pueden resolver problemas mediante el uso de los dispositivos móviles.

2-5. Los docentes saben discriminar adecuadamente la información relevante disponible en la red.

2-6. Los docentes saben producir contenido digital utilizando los dispositivos móviles. 


\section{Indicadores V3. Construcción del conocimiento}

3-1. Los estudiantes tienen nociones previas sobre el contenido.

3-2. Los estudiantes son capaces de generar nuevo conocimiento.

3-3. Los docentes actúan como guías y apoyo en el aprendizaje del estudiante.

3-4. Se establece un feedback entre el docente y el alumnado en las diferentes tareas.

Tabla 6. Indicadores de calidad asociados a la variable construcción del conocimiento

\begin{tabular}{|l|}
\hline \multicolumn{1}{|c|}{ Indicadores V4. Autorregulación del aprendizaje } \\
\hline 4-1. Los estudiantes se implican en su propio proceso de aprendizaje. \\
\hline 4-2. Las actividades favorecen la autorregulación del aprendizaje. \\
\hline 4-3. Los estudiantes son capaces de autorregular su aprendizaje. \\
\hline $\begin{array}{l}\text { 4-4. Los estudiantes consideran que el mobile learning les ayuda a autorregular su } \\
\text { aprendizaje. }\end{array}$ \\
\hline
\end{tabular}

Tabla 7. Indicadores de calidad asociados a la variable autorregulación del aprendizaje

\section{Indicadores V5. Trabajo cooperativo}

5-1. La disposición del mobiliario del aula favorece la cooperación entre los estudiantes.

5-2. Se forman grupos para trabajar cooperativamente.

5-3. Los grupos se forman de manera heterogénea.

5-4. Los estudiantes cooperan entre ellos.

5-5. Los docentes propician la cooperación entre los estudiantes.

5-6. Los docentes diseñan actividades que favorezcan el trabajo cooperativo.

\section{Discusión y conclusiones}

Los indicadores de calidad expuestos responden al interés y adecuación para evaluar diferentes elementos curriculares (Cardoso y Cerecedo, 2011) y en concreto las TIC (Rodríguez y Pantoja, 2014; Nolasco y Ojeda, 2016). En el caso de las variables "Dispositivos móviles", "Competencia digital” y "Trabajo cooperativo" concuerdan en sus características con las categorías establecidas en el Marco 
para el Análisis, Diseño y Evaluación de experiencias de m-learning (MADE-mlearn) establecido por Herrera, Sanz y Fennema (2013). Por otra parte, las variables "Autorregulación del aprendizaje" y "Trabajo cooperativo" coinciden con el modelo de buenas prácticas TIC de Fernández (2016).

En relación a la sugerencia sobre el indicador 1-1. Los estudiantes disponen de dispositivos móviles, aunque resulte redundante es imprescindible conocer si disponen de dispositivos móviles (Ramírez y García, 2017) para poder establecer el carácter individual o grupal de la actividad. Respecto al indicador 1-3. Los estudiantes utilizan únicamente los dispositivos móviles en el tiempo destinado a su aplicación en el aula, la actividad planteada debe ser motivante e incurrir en la autorregulación del aprendizaje (Suárez, Crescenzi y Grané, 2013) para que los estudiantes se focalicen en el desarrollo de la tarea. En cuanto al lenguaje en el que se expresa el indicador es entendible ya que no recae en vocablos específicos, cumpliendo así el principio de interpretabilidad (DANE, 2009).

Atendiendo a las sugerencias del indicador 1-4. Los docentes planifican los recursos a utilizar mediante los dispositivos móviles y establecen pautas para su uso, el término "control temporal" se encuentra implícito en la planificación, además este concepto puede generar confusión y ser por tanto contrario al criterio de interpretabilidad. En cuanto al indicador 1-5. Los docentes saben manejar adecuadamente los dispositivos móviles, se contextualiza que el manejo de los dispositivos se refiere a su aplicación didáctica en el aula. Por tanto, como comenta el experto, debe implicar el conocimiento y habilidad para su uso pedagógico.

Por otro lado, las consideraciones sobre la variable "Competencia digital (V2)" quedan recogidas en su definición y en los indicadores que la componen, los cuales responden a las áreas establecidas en el Marco Común de Competencia Digital Docente del Instituto Nacional de Tecnologías Educativas y Formación del Profesorado de España (INTEF, 2017) que sigue los preceptos del marco común europeo.

En torno a las sugerencias del indicador 2-1. Los estudiantes saben discriminar adecuadamente la información relevante disponible en la red, se incurriría en el incumplimiento del criterio de no redundancia (DANE, 2009) al haber dos indicadores que midan lo mismo, puesto que saber discriminar la información requiere de la búsqueda previa (INTEF, 2017). Del mismo modo el indicador 2-3. Los estudiantes son capaces de comunicar y compartir la información socialmente a través de los dispositivos móviles, no establece diferentes niveles de aplicación (académico, social, laboral), puesto que una buena práctica de mobile learning debe desarrollar la competencia digital (Ramos, Herrera y Ramírez, 2010) y esta abarca todos los ámbitos de la vida.

En base a las consideraciones sobre la variable "Construcción del conocimiento (V3)", la ubicuidad es una característica inherente al mobile learning (Brazuelo y Gallego, 2011) y resulta transversal en 
todas las variables, por lo que no requiere su inclusión específica en una de ellas. En cambio, en el indicador 3-2. Los estudiantes son capaces de generar nuevo conocimiento, se indica la necesidad de añadir "transferencia a la realidad", lo cual requeriría de otros instrumentos para medir y constatar esta variable, y, por tano, no ofrecería datos de forma inmediata y no cumpliría el criterio de oportunidad (DANE, 2009). Respecto al indicador 3-4. Se establece un feedback entre el docente y el alumnado en las diferentes tareas, se sugiere la inclusión del alumnado entre sí, aspecto que se recoge en la variable 5 (Trabajo cooperativo), por consiguiente incluirlo en este indicador incidiría en el hecho de generar dos indicadores que miden una misma cosa (criterio de no redundancia).

A propósito de la variable "Autorregulación del aprendizaje (V4)" se han efectuado los cambios sugeridos por el experto en base a las aportaciones de Suárez, Crescenzi y Grané (2013) y Sevillano y Vázquez (2015) sobre que el mobile learning favorece la autorregulación del aprendizaje. Del mismo modo se da respuesta a las apreciaciones sobre el indicador 4-4. Los estudiantes consideran que el mobile learning les ayuda a autorregular su aprendizaje, basándonos en la experiencia empírica de estos autores. Así pues, el indicador 4-2. Las actividades favorecen la autorregulación del aprendizaje, el término "actividad" engloba todo lo que concierne a las distintas tareas puestas en práctica con smartphones o tablets con la finalidad de que el alumnado sea capaz de autorregularse.

Por último, en la definición de trabajo cooperativo (V5) queda establecido su significado, de igual forma atendiendo a las sugerencias sobre el indicador 5-1. La disposición del mobiliario del aula favorece la cooperación entre los estudiantes, se ha matizado el término cooperación para no dar lugar a la confusión con colaboración.

Esta propuesta recoge el establecimiento de un sistema de indicadores de calidad para evaluar buenas prácticas docentes con mobile learning, totalmente necesario y requerido en el momento actual, en el que esta metodología docente está emergiendo. Se demanda contar con modelos de referencia como el aquí expuesto para discernir la simple introducción de los dispositivos móviles en el aula, del uso planificado y con carácter didáctico-pedagógico que debe tener el mobile learning. No obstante, los indicadores de calidad recogidos responden al sustento teórico que se ha consolidado en estos últimos años, así como a los resultados tangibles de las investigaciones llevadas a cabo, a lo que se suma la validación realizada por un grupo de expertos en la temática.

Es de resaltar el carácter innovador y la aportación que realiza este estudio al conocimiento en el campo de la evaluación de la calidad del mobile learning. Un campo incipiente y sin apenas investigación realizada, cuando debería ser el punto de partida para poder introducir con éxito una metodología emergente, como es el caso del mobile learning. Entre las principales aportaciones, destacamos que se trata de: (1) un sistema fundamentado y validado que sirve de referente para poner el foco de atención en las prácticas que se están llevando a cabo con dispositivos móviles en el aula y, en 
consecuencia, actua tanto como modelo para los investigadores en esta temática, como para los docentes que aplican esta metodología y no tienen en cuenta ciertas variables necesarias para sacar todo el potencial y rentabilidad al mobile learning; (2) un cuerpo sólido de conocimiento sobre las buenas prácticas en mobile learning, el cual puede servir de base para fundamentar nuevas propuestas e investigaciones en esta línea; (3) una acotación de las variables clave para que la introducción de los dispositivos móviles en el aprendizaje sea significativa y mejore el aprendizaje de los estudiantes.

Finalmente, como futuras líneas de investigación a raíz de este trabajo, nos encontramos, por un lado, con implicaciones de carácter teórico en base a la conversión de los indicadores en un instrumento de recogida de datos generalizable. Otras líneas, de carácter empírico, se avanzan desde la aplicación concreta de este sistema de indicadores en el aula universitaria, donde se verifique la existencia de los distintos indicadores en las prácticas de mobile learning, con el fin de discernir buenas prácticas docentes. También, se augura como foco de interés la recopilación de experiencias de éxito con dispositivos móviles.

\section{Referencias}

Aznar, I., Romero, J.M. y Rodríguez-García, A.M. (2018). La tecnología móvil de Realidad Virtual en educación: una revisión del estado de la literatura científica en España. EDMETIC, Revista de Educación Mediática y TIC, 7(1), 256-274. doi: https://doi.org/10.21071/edmetic.v7i1.10139

Brazuelo, F. y Gallego, D.J. (2011). Mobile Learning. Los dispositivos móviles como recurso educativo. Sevilla: MAD.

Cabero, J. y Barroso, J. (2013). La utilización del juicio de experto para la evaluación de TIC: el coeficiente de competencia experta. Bordón, 65(2), 25-38. doi: https://doi.org/10.13042/brp.2013.65202

Cabero, J. y Llorente, M.C. (2013). La aplicación del juicio de experto como técnica de evaluación de las tecnologías de la información (TIC). Eduweb. Revista de Tecnología de Información y Comunicación en Educación, 7(2), 11-22.

Caldeiro, M.C., Yot, C. y Castro, A. (2018). Detección de buenas prácticas docentes de uso de dispositivos móviles en primaria a través del análisis documental. Prisma Social, 20, 58-75.

Cardoso, E. y Cerecedo, M. (2011). Propuesta de indicadores para evaluar la calidad de un programa de posgrado en Educación. Revista Electrónica de Investigación Educativa, 13(2), 68-82. 
Departamento Administrativo Nacional de Estadística de Colombia (DANE) (2009). Guía para Diseño, Construcción e Interpretación de Indicadores. Colombia: Dirección de Difusión, Mercadeo y Cultura Estadística.

Escobar, J. y Cuervo, A. (2008). Validez de contenido y juicio de expertos: una aproximación a su utilización. Avances en medición, 6(1), 27-36.

Fernández, B. (2018). La utilización de objetos de aprendizaje de realidad aumentada en la enseñanza universitaria de educación primaria. International Journal of Educational Research and Innovation (IJERI), 9, 90-104.

Fernández, J.M. y Torres, J.A. (2015). Actitudes docentes y buenas prácticas con TIC del profesorado de Educación Permanente de Adultos en Andalucía. Revista Complutense de Educación, 26, 33-49. doi: https://doi.org/10.5209/rev_RCED.2015.v26.43812

Fernández, M.D. (2016). Modelo educativo emergente en las buenas prácticas TIC. Revista Fuentes, 18(1), 33-47. doi: https://doi.org/10.12795/revistafuentes.2016.18.1.02

García-Valcárcel, A. y Tejedor, F.J. (2010). Evaluación de procesos de innovación escolar basados en el uso de las TIC desarrollados en la Comunidad de Castilla y León. Revista de Educación, 352, 125-147.

Gutiérrez, A., Rodríguez, A.E. y Pantoja, M. (2014). Evaluación del uso de las TIC en Educación para el Desarrollo. Obtención de indicadores de buenas prácticas mediante análisis factorial. RED - Revista de Educación a Distancia, 41, 1-37.

Herrera, S., Sanz, C. y Fennema, C. (2013). MADE-mlearn: un marco para el análisis, diseño y evaluación de experiencias de m-learning en el nivel de postgrado. TE\&ET, 10, 7-15

Instituto Nacional de Tecnologías Educativas y de Formación del Profesorado de España (INTEF) (2017). Marco Común de Competencia Digital Docente. España: Ministerio de Educación, Cultura y Deporte.

Jeno, L., Grytnes, J. y Vandvik, V. (2017). The effect of a mobile-application tool on biology students' motivation and achievement in species identification: A Self-Determination Theory perspective. Computers \& Education, 107, 1-12. doi: https://doi.org/10.1016/j.compedu.2016.12.011

Kearney, M., Schuck, S., Burden, K. y Aubusson, P. (2012). Viewing Mobile Learning from a Pedagogical Perspective. Research in Learning Technology, 20(1), 1-17. doi: https://doi.org/10.3402/rlt.v20i0.14406 
Mateus, J.C., Aran-Ramspott, S. y Masanet, M.J. (2017). Análisis de la Literatura sobre Dispositivos Móviles en la Universidad Española. RIED. Revista Iberoamericana de Educación a Distancia, 20(2), 4972. doi: https://doi.org/10.5944/ried.20.2.17710

Monguillot, M., González, C., Guitert, M. y Zurita, C. (2014). Mobile learning: una experiencia colaborativa mediante códigos QR. RUSC, 11(1), 175-191.

Nolasco, P. y Ojeda, M.M. (2016). La evaluación de la integración de las TIC en la educación superior: fundamento para una metodología. RED - Revista de Educación a Distancia, 48, 1-24. doi: https://doi. org/10.6018/red/48/9

Ramírez-Montoya, M.S. y García-Peñalvo, F.J. (2017). La integración efectiva del dispositivo móvil en la educación y en el aprendizaje. RIED. Revista Iberoamericana de Educación a Distancia, 20(2), 29-47. doi: https://doi.org/10.5944/ried.20.2.18884

Ramos, A.I., Herrera, J.A. y Ramírez, M.S. (2010). Desarrollo de habilidades cognitivas con aprendizaje móvil: un estudio de casos. Comunicar, 17(34), 201-209. doi: https://doi.org/10.3916/C34-2010-03-20 Robles, P. y Rojas, M.C. (2015). La validación por juicio de expertos: dos investigaciones cualitativas en Lingüística aplicada. Revista Nebrija de Lingüística Aplicada, 18, 1-16.

Sevillano, M.L. y Vázquez, E. (2015). The Impact of Digital Mobile Devices in Higher Education. Educational Technology \& Society, 18(1), 106-118.

Suárez, C., Lloret, C. y Mengual, S. (2016). Percepción docente sobre la transformación digital del aula a través de tabletas: un estudio en el contexto español. Comunicar, 24(49), 81-89. doi: https://doi. org/10.3916/C49-2016-08

Suárez, R., Crescenzi, L. y Grané, M. (2013). Análisis del entorno colaborativo creado para una experiencia de mobile learning. Education in the Knowledge Society, 14(1), 101-122.

Wu, W.H., Wu, Y.C.J., Chen, C.Y., Kao, H.Y., Lin, C.H. y Huang, S.H. (2012). Review of trends from mobile learning studies: A meta-analysis. Computers \& Education, 59(2), 817-827. doi: https://doi. org/10.1016/j.compedu.2012.03.016 